\title{
The Long-term Clinical Course of Chronic Eosinophilic Pneumonia
}

\author{
Takashi Ishiguro ${ }^{1}$, Noboru Takayanagi ${ }^{1}$, Ryuji Uozumi ${ }^{2}$, Mami Tada ${ }^{1}$, Naho Kagiyama ${ }^{1}$, \\ Yotaro Takaku ${ }^{1}$, Yoshihiko Shimizu ${ }^{3}$, Yutaka Sugita ${ }^{1}$ and Satoshi Morita ${ }^{2}$
}

\begin{abstract}
Objective The long-term clinical course and prognosis of patients with chronic eosinophilic pneumonia (CEP) including factors predictive of the relapse of CEP have not been fully investigated. The aim of the present study was to investigate these issues.

Methods We retrospectively investigated the rate of relapse and prognosis in 73 patients diagnosed as having CEP.

Results Systemic corticosteroid therapy was administered at a prednisolone dose of $29.4 \pm 7.6 \mathrm{mg} / \mathrm{day}$. During a median follow-up period of 1,939 days, 27 patients suffered from relapse of CEP. Two patients developed steroid-induced diabetes mellitus, and 1 patient developed pulmonary nontuberculous mycobacteriosis. Five patients died; however, none died of CEP. A history of smoking was the only independent negative risk factor for relapse of CEP [hazard ratio, 0.37 (0.14-0.98)].

Conclusion Patients with CEP frequently relapse. During the follow-up, metabolic and infectious complications under prolonged corticosteroid therapy are problematic. A history of smoking was a negative factor for predicting the risk of CEP relapse.
\end{abstract}

Key words: chronic eosinophilic pneumonia, relapse, smoking, prognosis

(Intern Med 55: 2373-2377, 2016)

(DOI: 10.2169/internalmedicine.55.6765)

\section{Introduction}

Chronic eosinophilic pneumonia (CEP), which was first individualized as an entity by Carrington et al. (1), is characterized by the idiopathic, progressive onset of symptoms leading within a few weeks to an infiltrative pulmonary disease with cough, increasing dyspnea, malaise, and weight loss. A dramatic response to corticosteroid treatment is generally observed in CEP; however, most patients require prolonged treatment. In one series, CEP recurred in $58 \%$ of patients after corticosteroids were discontinued, and $21 \%$ of the patient relapsed while corticosteroids were being tapered (2). In another series, relapses occurred in half of the patients after corticosteroids were weaned or tapered (3).

There is also a concern that prolonged treatment with cor- ticosteroid will induce diabetes mellitus (DM) or infections; however, these complications were not covered in previous reports assessing the long-term follow-up of CEP patients (2-6). In addition, to our knowledge, only one report has assessed the predictive factors of CEP relapse, and to date, it is not known which factors are predictive of relapse of CEP (7). Thus, the aim of the present study was to investigate the frequency at which patients with CEP developed DM or infections, focusing especially on pulmonary nontuberculous mycobacteriosis (pNTM), of which corticosteroid therapy is a risk factor (8), and to assess factors predictive of the relapse of CEP.

\section{Materials and Methods}

We performed a retrospective study of 73 patients hospi-

\footnotetext{
${ }^{1}$ Department of Respiratory Medicine, Saitama Cardiovascular and Respiratory Center, Japan, ${ }^{2}$ Department of Biomedical Statistics and Bioinformatics, Kyoto University Graduate School of Medicine, Japan and ${ }^{3}$ Department of Pathology, Saitama Cardiovascular and Respiratory Center, Japan

Received for publication October 29, 2015; Accepted for publication January 5, 2016

Correspondence to Dr. Takashi Ishiguro, ishiguro.takashi@pref.saitama.lg.jp
} 
Table 1. Characteristics of Patients with Chronic Eosinophilic Pneumonia (n=73).

\begin{tabular}{lcc}
\hline Characteristic & Value & $(\%)$ \\
\hline Age (years) & $56.7 \pm 12.6$ & \\
Male sex & 26 & $(35.6)$ \\
Smoking history & 25 & $(34.2)$ \\
Underlying diseases & & \\
Asthma & 35 & $(47.9)$ \\
COPD & 4 & $(5.5)$ \\
Bronchiectasis & 0 & $(0)$ \\
pNTM & 2 & $(2.7)$ \\
Old TB & 3 & $(4.1)$ \\
Pollenosis, allergic rhinitis, & 11 & $(15.1)$ \\
sinusitis, atopic dermatitis & & \\
Gastric ulcer, duodenal ulcer & 3 & $(4.1)$ \\
Congestive heart failure & 1 & $(1.4)$ \\
DM & 6 & $(8.2)$ \\
Hypertension & 11 & $(15.81)$ \\
None & 20 & $(27.4)$ \\
Treatment & & \\
Systemic corticosteroid therapy & 65 & $(89.0)$ \\
Follow-up period (days) & $1,939(18-10,405)$ & \\
Relapse & 27 & $(37.0)$ \\
Developed DM & 2 & $(2.7)$ \\
Developed pNTM & 1 & $(1.4)$ \\
Death & 5 & $(6.6)$ \\
\hline COPD: chronic obstructive pulmonary & disease, DM: & diabetes \\
mellitus, pNTM: pulmonary non-tuberculous mycobacteriosis, TB: \\
tuberculosis. Values are mean \pm SD, median (range), or number $(\%)$.
\end{tabular}

talized with CEP at our institution in Saitama, Japan. With reference to previous reports $(6,9,10)$ analyzing the characteristics of CEP, diagnosis of CEP was based on a greater than 2-month history of symptoms prior to diagnosis and fulfilment of at least one of the following criteria: (i) histological diagnosis by surgical or transbronchial lung biopsy, (ii) bronchoalveolar lavage fluid (BALF) eosinophilia $>15 \%$, and (iii) fulfilment of two of criteria (a)-(c): (a) peripheral blood eosinophilia $\left(>0.5 \times 10^{9} / \mathrm{L}\right)$, (b) BALF eosinophilia $>10 \%$, or (c) increase in eosinophils in lung tissue obtained via transbronchial lung biopsy $(6,9)$. Serum $\operatorname{IgE}$ or $\operatorname{IgG}$ against several fungi was investigated via radioallergosorbent test, enzyme-linked immunoassay, and complement fixation method.

We excluded patients who were diagnosed on development of CEP as having eosinophilic granulomatosis with polyangiitis, allergic bronchopulmonary mycosis, acute eosinophilic pneumonia (EP), drug-induced EP, EP due to parasite infection, desquamative interstitial pneumonia, or Langerhans cell histiocytosis at the time of EP diagnosis or during follow-up.

Relapse of CEP was defined as the development of new shadows on chest X-ray, but which excluded new shadows resulting from infection or any other causes. Peripheral blood eosinophilia and development of respiratory symptoms without the development of new shadows on chest X-ray were not regarded as relapse of CEP.

\section{Statistical analysis}

All data are expressed as mean \pm SD or median (range) for continuous variables and frequency (percentage) for categorical variables unless otherwise specified. A Student $t$-test
Table 2. Symptoms Associated with the Development of Chronic Eosinophilic Pneumonia.

\begin{tabular}{lcc}
\hline & $\mathrm{n}$ & $(\%)$ \\
\hline Cough & 54 & $(73.9)$ \\
Sputum & 27 & $(37.0)$ \\
Hemosputum & 1 & $(1.4)$ \\
Appetite loss & 2 & $(2.7)$ \\
General fatigue & 5 & $(6.9)$ \\
Dyspnea & 27 & $(37.0)$ \\
Low-grade fever, fever & 29 & $(39.7)$ \\
Rales & 16 & $(21.9)$ \\
Body weight loss & 5 & $(6.9)$ \\
Chest pain, discomfort & 3 & $(4.1)$ \\
Abdominal pain & 0 & $(0)$ \\
Skin rash & 0 & $(0)$ \\
Neurological symptoms, muscle weakness & 0 & $(0)$ \\
None & 2 & $(2.7)$ \\
\hline
\end{tabular}

was performed to compare the groups with or without a history of smoking in relation to the daily dose of prednisolone (PSL) at the initial relapse of CEP. The Satterthwaite $t$-test was also used to compare the dose of PSL between groups with or without relapse of CEP. The cumulative relapse rate of CEP was calculated using the Kaplan-Meier method, and the log-rank test was used to compare the relapse rate. Twosided $95 \%$ confidence intervals of the relapse rate were constructed using a log-log transformation. The Cox proportional hazards model was used to derive a hazard ratio and two-sided $95 \%$ confidence interval for univariate and multivariate analyses. A p value $<0.05$ was regarded to be statistically significant. All statistical analyses were performed with the SAS version 9.3 (SAS Institute, Cary, USA).

Approval for this study was obtained from the institutional clinical research ethics board (approval no. 2015019; August 5, 2015).

\section{Results}

The mean patient age was $56.7 \pm 12.6$ years, and 26 $(35.6 \%)$ patients were men (Table 1). Of the 73 patients studied, 25 (34.2\%) had a history of smoking [all were current smokers with a median (range) of 21.3 (3.5-120) packyears, and 16 were men], and $5(6.8 \%)$ had a history of exposure to dust. Underlying diseases in the 73 patients included asthma in 35 (47.9\%), allergic disorder [pollinosis, atopic dermatitis, allergic rhinitis (3 of whom also had asthma), and sinusitis] in $11(15.1 \%)$ patients, chronic obstructive pulmonary disease in $4(5.5 \%)$, old tuberculosis in $3(4.1 \%)$, pNTM in $2(2.7 \%)$, gastrointestinal ulcer in 3 (4.1\%), DM in $6(8.2 \%)$, hypertension in $11(15.1 \%)$, and congestive heart failure in 1 (1.4\%). Symptoms found on presentation are listed in Table 2.

Pulmonary function tests were performed in 49 patients (67.1\%) on presentation. Twenty-nine patients showed restrictive ventilatory impairment (predicted vital capacity $<80 \%$ ), and 23 showed airflow limitation (forced expiratory volume in 1 second/forced vital capacity ratio $<70 \%$ ).

Laboratory findings on admission are listed in Table 3. Blood test values included an eosinophil count of 2,065 \pm 
Table 3. Laboratory Findings of Patients with Chronic Eosinophilic Pneumonia.

\begin{tabular}{|c|c|c|}
\hline & $\mathrm{n}$ or mean $\pm \mathrm{SD}$ & Median (range) \\
\hline White blood cell count $\left(/ \mathrm{mm}^{3}\right)$ & $9,931 \pm 3,464$ & $9,500(4,900-24,300)$ \\
\hline Eosinophils $\left(/ \mathrm{mm}^{3}\right)$ & $2,065 \pm 2,095$ & $1,500(730-11,200)$ \\
\hline Eosinophils (\%) & $19.4 \pm 14.6$ & $16.6(10.8-64.2)$ \\
\hline C-reactive protein $(\mathrm{mg} / \mathrm{dL})$ & $5.0 \pm 6.4$ & $2.5(0-38.4)$ \\
\hline Serum $\operatorname{IgE}(\mathrm{IU} / \mathrm{mL})$ & $620 \pm 714$ & $316(37-3,029)$ \\
\hline \multicolumn{3}{|l|}{ Bronchoalveolar lavage fluid } \\
\hline Eosinophils (\%) & $41.4 \pm 28.3$ & $34(4-93)$ \\
\hline $\mathrm{CD} 4 / 8$ ratio & $2.1 \pm 2.0$ & $1.8(0.4-13.2)$ \\
\hline \multicolumn{3}{|c|}{ Serum-specific IgE (positive/tested) } \\
\hline Aspergillus sp. & $10 / 52$ & \\
\hline Candida $\mathrm{sp}$. & $6 / 52$ & \\
\hline Penicillium sp. & $5 / 52$ & \\
\hline Cladosporium sp. & $1 / 52$ & \\
\hline Alternaria $\mathrm{sp}$. & $1 / 52$ & \\
\hline Schizophyllum commune & $9 / 19$ & \\
\hline \multicolumn{3}{|c|}{ Serum-specific IgG (positive/tested) } \\
\hline Aspergillus sp. & $0 / 52$ & \\
\hline Schizophyllum commune & $2 / 52$ & \\
\hline
\end{tabular}

Table 4. Prednisolone Dose at Final Follow-up in Patients without Relapse of CEP and on Relapse in Patients with Relapse of CEP.

\begin{tabular}{lccc}
\hline & $\begin{array}{c}\text { Dose in patients } \\
\text { without relapse of } \\
\operatorname{CEP}(\mathrm{n}=46)^{1}(\%)\end{array}$ & $\begin{array}{c}\text { Dose in patients } \\
\text { with relapse of }\end{array}$ & pEP value $(\mathrm{n}=27)^{2}(\%)$
\end{tabular}

2,095 [median, 1,500 (730-11,200)]/ $\mathrm{mm}^{3}$, C-reactive protein of $5.0 \pm 6.4$ [median, $2.5(0-38.4)] \mathrm{mg} / \mathrm{dL}$, and immunoglobulin $\mathrm{E}$ of $620 \pm 714$ [median, 316 (37-3,029)] IU/mL. Respiratory failure (defined as a $\mathrm{PaO}_{2}<60$ Torr under ambient air) occurred in $10(13.7 \%)$ patients.

Chest X-ray findings were compatible with those reported in CEP $(11,12)$, which were characterized by the presence of homogeneous peripheral airspace consolidation. Computed tomography findings characterized by consolidation and frequent peripheral and patchy distribution, patchy or peripheral ground-glass opacities, and the occasional association with crazy paving, were also compatible with those reported previously (13).

Systemic corticosteroid therapy was administered to 65 patients, with a mean dose of PSL of $29.4 \pm 7.6 \mathrm{mg} /$ day $(0.60$ $\pm 0.16 \mathrm{mg} / \mathrm{kg} / \mathrm{day})$. Corticosteroid therapy was not administered in 8 patients because their lung shadows had spontaneously disappeared. Cessation of smoking was required after admission for all patients with a history of smoking.

The median follow-up period was 1,939 (range, 18$10,405)$ days. During follow-up, smoking cessation was confirmed in all patients by physicians during outpatient visits. Two of the 65 patients who received systemic corticosteroid therapy developed steroid-induced DM, and diabetes control

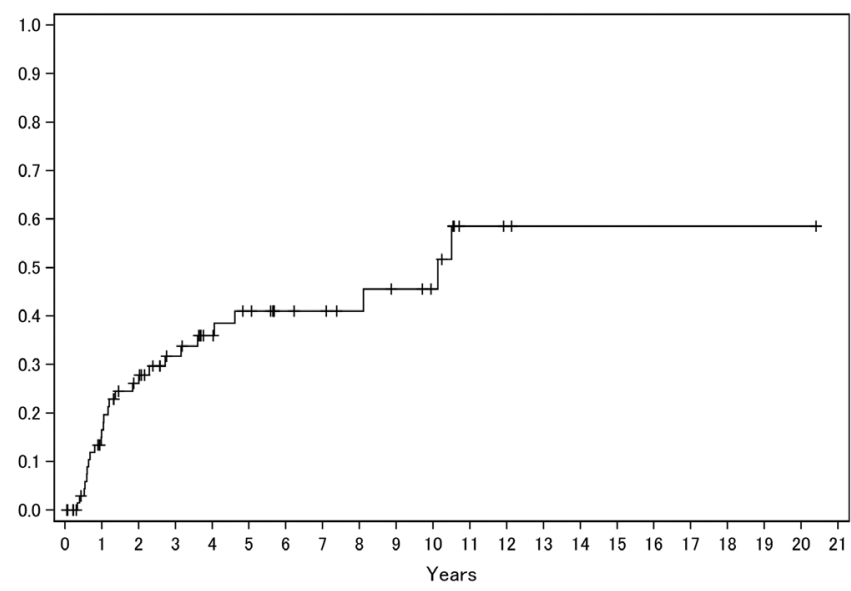

Figure 1. Kaplan-Meier curve for the relapse rate of chronic eosinophilic pneumonia (CEP). Overall, the annual relapse rate $(95 \%$ confidence interval) of CEP was $0.1654(0.0952$, 0.2789). $X$ axis: years after the diagnosis of CEP, $Y$ axis: the cumulative relapse rate.

in 2 other patients who already had DM before starting systemic corticosteroid therapy worsened and these patients required insulin therapy. Another patient developed pNTM. Twenty-seven (37.0\%) patients suffered from relapse of CEP. The cumulative annual relapse rate was 0.1654

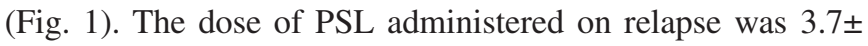
$4.1 \mathrm{mg} /$ day, median $3 \mathrm{mg} /$ day $(0-17.5 \mathrm{mg} /$ day $)$ and did not differ significantly from that being administered at final follow-up in the patients without relapse (Table 4). The number of patients on maintenance corticosteroid therapy without relapse tended to be higher than that of patients with relapse, but the difference was not significant $(\mathrm{p}=$ 0.057). After excluding 1 patient in whom CEP relapsed 10 years after PSL cessation, the median duration between PSL cessation and relapse of CEP was 56 (28-191) days. A Cox proportional hazards model analysis showed a history of smoking to be the only independent risk factor of relapse of CEP by multivariate analysis (Table 5, Fig. 1,2). Other factors on presentation, including age ( $\geq 65$ years old), sex, history of dust exposure, underlying disorders (asthma and allergic disorders), respiratory failure, peripheral eosinophilia $\left(>1,000 / \mathrm{mm}^{3}\right.$ or $>30 \%$ of white blood cells), serum IgE level $(>1,000 \mathrm{IU} / \mathrm{mL})$, rate of eosinophils in the BALF $(>50 \%)$, CD4/8 ratio in the BALF $(<1$ or $\geq 3)$, and inhaled steroid therapy, were not significant predictive factors by univariate analysis. The daily dose of PSL on the initial relapse of CEP was $1.7 \pm 2.0 \mathrm{mg}$ in patients with a history of smoking, whereas it was $4.3 \pm 4.1 \mathrm{mg}$ in patients without a history of smoking, but the difference between the two groups was not significantly ( $\mathrm{p}=0.184$, Student $t$-test).

During follow-up, 5 patients died, but none died from CEP. The 68 surviving patients have shown good functional status, their respiratory condition has remained stable; and none have required long-term oxygen therapy. Corticosteroid therapy was not necessary in $37(50.7 \%)$ of the 73 patients at the final follow-up. 
Table 5. Univariate and Multivariate Analysis for Predictive Factors of Chronic Eosinophilic Pneumonia.

\begin{tabular}{|c|c|c|c|c|}
\hline \multirow[b]{2}{*}{ Factor } & \multicolumn{2}{|c|}{ Univariate analysis } & \multicolumn{2}{|c|}{ Multivariate analysis } \\
\hline & $\begin{array}{c}\text { HR } \\
(95 \% \mathrm{CI}) \\
\end{array}$ & $\mathrm{p}$ value & $\begin{array}{c}\text { HR } \\
(95 \% \mathrm{CI}) \\
\end{array}$ & $\mathrm{p}$ value \\
\hline Age $(\geq 65$ years old $)$ & $0.76(0.29,2.00)$ & 0.575 & & \\
\hline Male sex & $0.91(0.40,2.08)$ & 0.816 & & \\
\hline Current smoking, yes & $0.41(0.16,1.01)$ & 0.045 & $0.37(0.14,0.98)$ & 0.045 \\
\hline Dust exposure, yes & $0.40(0.05,2.95)$ & 0.352 & & \\
\hline Pollenosis, allergic rhinitis, sinusitis, atopic & $0.77(0.27,2.24)$ & 0.635 & & \\
\hline Respiratory failure on presentation & $0.37(0.05,2.71)$ & 0.305 & & \\
\hline Peripheral blood eosinophilia $\left(\geq 1,000 / \mathrm{mm}^{3}\right)$ & $0.98(0.45,2.11)$ & 0.956 & $0.73(0.31,1.74)$ & 0.480 \\
\hline Peripheral blood eosinophilia ( $\geq 30 \%$ ) & $0.57(0.22,1.52)$ & 0.256 & & \\
\hline Elevated C-reactive protein $(\geq 10 \mathrm{mg} / \mathrm{dL})$ & $1.38(0.47,4.01)$ & 0.556 & & \\
\hline Elevated serum IgE level $(\geq 1,000 \mathrm{IU} / \mathrm{mL})$ & $0.55(0.17,1.84)$ & 0.326 & & \\
\hline Positive IgE or IgG antibody against fungi & $1.11(0.50,2.48)$ & 0.791 & $1.42(0.55,3.63)$ & 0.468 \\
\hline BALF lymphocytes $\geq 30 \%$ & $0.65(0.24,1.76)$ & 0.396 & & \\
\hline BALF eosinophils $\geq 50 \%$ & $1.50(0.67,3.32)$ & 0.319 & $1.53(0.64,3.68)$ & 0.342 \\
\hline $\mathrm{CD} 4 / 8$ ratio $\geq 3$ & $1.59(0.60,4.21)$ & 0.347 & & \\
\hline $\mathrm{CD} 4 / 8$ ratio $<1$ & $1.73(0.79,3.79)$ & 0.163 & $1.79(0.80,3.97)$ & 0.155 \\
\hline Systemic steroid therapy, yes & $3.78(0.51,27.91)$ & 0.161 & & \\
\hline Inhaled steroid therapy, yes & $0.72(0.32,1.62)$ & 0.432 & & \\
\hline
\end{tabular}

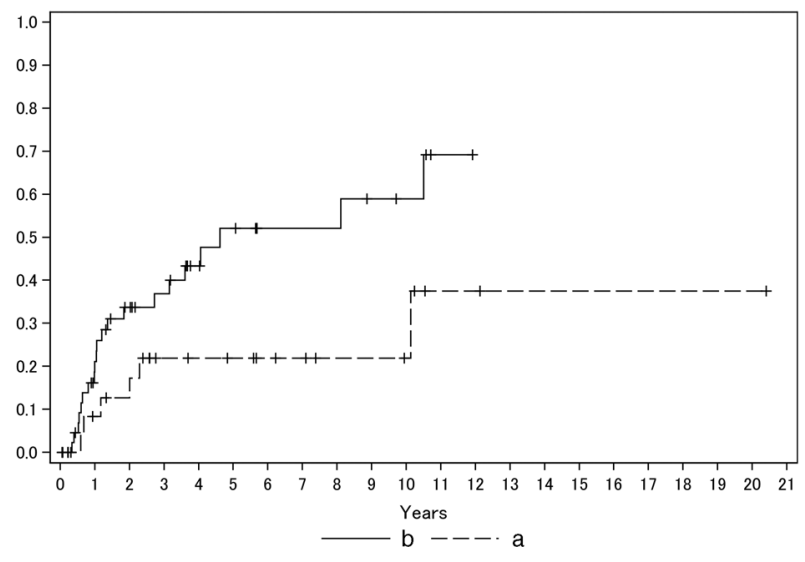

Figure 2. Kaplan-Meier curves for the relapse rate of chronic eosinophilic pneumonia (CEP). The annual relapse rate (95\% confidence interval) of CEP was $0.0833(0.0215,0.2939)$ in patients who were smokers (a) at the diagnosis of CEP, whereas the rate was $0.2112(0.1158,0.3670)$ in patients who were nonsmokers at the diagnosis of $\operatorname{CEP}(b) . X$ axis: years after the diagnosis of CEP, $Y$ axis: the cumulative relapse rate.

\section{Discussion}

We herein investigated the clinical course of 73 patients who were diagnosed as having CEP on their first presentation. During follow-up, 2 of the 65 patients who received systemic corticosteroid therapy developed DM. Twentyseven patients suffered a relapse of CEP. Five of 73 patients died of diseases other than CEP. A history of smoking at the diagnosis of CEP was the only a negative predictive factor for relapse of CEP.

Although a favorable response to systemic corticosteroid therapy was found and the initial PSL dose was sufficient at a median dose of $0.6 \mathrm{mg} / \mathrm{kg} / \mathrm{day}$, as recommended in previous reports (14), 27 of 73 patients relapsed during follow- up, which appears problematic. Marchand et al. (3) investigated the relapse ratio in 62 patients with CEP. Thirty patients suffered 48 relapses of EP, with relapse in 28 occurring an average of 55 weeks after cessation of corticosteroid therapy, and 20 relapsing on a daily PSL dose of about 10 mg. In contrast, $27(37.0 \%)$ of our patients relapsed on a PSL dose of $3.7 \pm 4.1 \mathrm{mg} /$ day. These differences may be due to physique (body weight), which is often lower in Asian people, but the exact body weight on relapse in many of our patients was unknown. CEP relapse occurred after PSL cessation at a median duration of 56 days, which was shorter than that reported by Marchand et al. (3) or Oyama et al. (7), but the exact reason remains unclear.

Although corticosteroid therapy remains a mainstay of CEP treatment, prolonged corticosteroid administration may induce metabolic and infectious complications. Two of our patients developed DM and diabetes control worsened in 2 other patients who already had DM before starting systemic corticosteroid therapy. Future studies are needed to clarify the effects of prolonged corticosteroid therapy on the cardiovascular system in CEP patients. One additional patient developed pNTM, for which corticosteroid is a risk factor. The limited number of patients with pNTM did not allow us to assess whether the incidence of pNTM was higher in our CEP patients than that already reported (15). Similar to the findings in other studies, although no deaths occurred from CEP, metabolic and infectious complications such as DM under prolonged corticosteroid therapy in patients with CEP are problematic in terms of prognosis. Additional treatment options are expected in the future. Because corticosteroid therapy was not necessary in about half of our patients at final follow-up, physicians should consider tapering and ending systemic corticosteroid treatment.

A history of smoking was a predictive factor for relapse of CEP at the time of initial diagnosis. Previously, Oyama et al. assessed this factor in 44 patients with CEP in a prospec- 
tive study (7), which showed that none of the investigated factors were significantly associated with relapse. To our knowledge, no other reports have assessed the predictive factors of CEP relapse. One possible explanation of our results is the contribution of smoking to the development or maintenance of eosinophilic infiltration in the lung (16). Although our understanding of the pathophysiology of CEP is incomplete, studies of BAL fluid from patients with CEP show elevations of several cytokine, chemokine, and immunomodulatory products (17-19), which suggests that a multifactorial process is involved in the recruitment of eosinophils into the lung (20). Although no reports have suggested an association between smoking and CEP, there is significant overlap between CEP and acute EP, for which a strong association exists with cigarette smoking (20), in the presence of specific common inflammatory mediators in the BALF (20). Smoking causes cellular and molecular changes that can lead to inflammation. In response to smoking, inflammatory signaling is altered by airway epithelial cells and macrophages, which, according to the signaling pathway used, results in an increase of lymphocytes, eosinophils, neutrophils, and mast cells in the lungs. In response to the exposure to smoking, a number of proteins are upregulated and secreted, many of which have immunomodulatory activities that contribute to the pathogenesis of disease (21). The inflammatory process induced by smoking is halted by its cessation, and all of our patients stopped smoking after the diagnosis of CEP, which may have helped to prevent the relapse of CEP. Large, prospective studies will be needed in the future to clarify this matter.

\section{Conclusion}

CEP frequently relapses, and a history of smoking in patients at the time of the initial diagnosis of CEP was found to be a negative predictive factor for relapse of CEP. There may be some population of CEP patients in whom smoking contributes to the development or maintenance of CEP. The relapse of CEP may be halted by smoking cessation, which is strongly suggested in CEP patients. Prolonged systemic corticosteroid therapy can increase metabolic or infectious complications. Additionally, because some patients do not require maintenance corticosteroid therapy, physicians should consider tapering and discontinuing systemic corticosteroid treatment.

\section{The authors state that they have no Conflict of Interest (COI).}

\section{Acknowledgement}

We thank Katsuhiko Kamei at the Medical Mycology Research Center, Chiba University, for measuring specific IgE and IgG antibodies against Schizophyllum commune.

\section{References}

1. Carrington $\mathrm{CB}$, Addington WW, Goff AM, et al. Chronic eosinophilic pneumonia. N Engl J Med 280: 787-798, 1969.

2. Jederlinic PJ, Sicilian L, Gaensler EA. Chronic eosinophilic pneumonia. A report of 19 cases and a review of the literature. Medicine (Baltimore) 67: 154-162, 1988.

3. Marchand E, Reynaud-Gaubert M, Lauque D, Durieu J, Tonnel $\mathrm{AB}$, Cordier JF. Idiopathic chronic eosinophilic pneumonia. A clinical and follow-up study of 62 cases. The Groupe d'Etudes et de Recherche sur les Maladies "Orphelines" Pulmonaires (GERM“O”P). Medicine (Baltimore) 77: 299-312, 1998.

4. Naughton M, Fahy J, FitzGerald MX. Chronic eosinophilic pneumonia. A long-term follow-up of 12 patients. Chest 103: 162-165, 1993.

5. Pearson DJ, Rosenow EC 3rd. Chronic eosinophilic pneumonia (Carrington's): a follow-up study. Mayo Clin Proc 53: 73-78, 1978.

6. Mochizuki Y, Kobashi Y, Nakahara Y, et al. Chronic eosinophilic pneumonia: a follow-up study of 12 cases. Nihon Kokyuki Gakkai Zasshi 40: 851-855, 2002 (in Japanese, Abstract in English).

7. Oyama Y, Fujisawa T, Hashimoto D, et al. Efficacy of short-term prednisolone treatment in patients with chronic eosinophilic pneumonia. Eur Respir J 45: 1624-1631, 2015.

8. Dirac MA, Horan KL, Doody DR, et al. Environment or host?: a case-control study of risk factors for Mycobacterium avium complex lung disease. Am J Respir Crit Care Med 186: 684-691, 2012.

9. Umeki S. Reevaluation of eosinophilic pneumonia and its diagnostic criteria. Arch Intern Med 152: 1913-1919, 1992.

10. Al-Jahdali H, Waness A, Al-Jawder S, et al. Eosinophilic pneumonia: experience at two tertiary care referral hospitals in Saudi Arabia. Ann Saudi Med 32: 32-36, 2012.

11. Jeong YJ, Kim KI, Seo IJ, et al. Eosinophilic lung diseases: a clinical, radiologic, and pathologic overview. Radiographics 27: 617-637, 2007.

12. Rossi G, Tironi A, Dore R, et al. Pulmonary eosinophilic infiltrates. Pathologica 102: 537-546, 2010.

13. Hypersensitivity pneumonitis and eosinophilic lung diseases. In: High-resolution CT of the Lung. 5th ed. Webb WR, Müller NL, Naidich DP, Eds. Wolters Kluwer Health, Philadelphia, 2015: 376396.

14. Marchand E, Cordier JF. Idiopathic chronic eosinophilic pneumonia. Orphanet J Rare Dis 1: 11, 2006.

15. Sakatani M. The non-tuberculous mycobacteriosis. Kekkaku 80: 25-30, 2005 (in Japanese, Abstract in English).

16. Botelho FM, Llop-Guevara A, Trimble NJ, et al. Cigarette smoke differentially affects eosinophilia and remodeling in a model of house dust mite asthma. Am J Respir Cell Mol Biol 45: 753-760, 2011.

17. Katoh S, Matsumoto K, Fukushima K, et al. Elevated chemokine levels in bronchoalveolar lavage fluid of patients with eosinophilic pneumonia. J Allergy Clin Immunol 58: 518-523, 2000.

18. Kita H, Sur S, Hunt LW, et al. Cytokine production at the site of disease in chronic eosinophilic pneumonitis. Am J Respir Crit Care Med 153: 1437-1441, 1996.

19. Miyazaki E, Nureki S, Fukami T, et al. Elevated levels of thymusand activation-regulated chemokine in bronchoalveolar lavage fluid from patients with eosinophilic pneumonia. Am J Respir Crit Care Med 165: 1125-1131, 2002.

20. Akuthota P, Weller PF. Eosinophilic pneumonias. Clin Microbiol Rev 25: 649-660, 2012.

21. Crotty Alexander LE, Shin S, Hwang JH. Inflammatory diseases of the lung induced by conventional cigarette smoke. Chest 148: 1307-1322, 2015.

The Internal Medicine is an Open Access article distributed under the Creative Commons Attribution-NonCommercial-NoDerivatives 4.0 International License. To view the details of this license, please visit (https://creativecommons.org/licenses/ by-nc-nd/4.0/).

(C) 2016 The Japanese Society of Internal Medicine

http://www.naika.or.jp/imonline/index.html 